\title{
Efecto de la intervención sobre los golpes de fondo por medio de un entrenamiento de 8 semanas de mini tennis
}

\author{
Ávila Anna Fitzpatrick, Keith Davids y Joseph Antony Stone \\ Universidad de Sheffield Hallam, Inglaterra
}

RESUMEN

La evidencia sugiere que las versiones modificadas del tenis (ej. LTA mini tennis) inciden positivamente sobre el desarrollo táctico y técnico de los niños. Sin embargo, Fitzpatrick, Davids y Stone (2017) destacaron que el mini tennis puede que sea menos conveniente para que los niños desarrollen su golpe de revés en comparación con el de derecha, lo que podría llevar a un desequilibrio de sus habilidades. Hemos investigado el efecto de la intervención de un entrenamiento de 8 semanas, diseñado para disminuir el desequilibrio entre el golpe de derecha y el de revés, en los partidos y el rendimiento de los niños (Fitzpatrick, Davids y Stone 2018). Tras la intervención, el grupo experimental realizó un porcentaje más alto de golpes de revés que el grupo control durante la disputa de partidos. El grupo experimental también demostró mejoras superiores en la eficiencia técnica del golpe de derecha y revés, comparado con el grupo control, y su habilidad de mantener el peloteo con el entrenador. Los resultados indican que las modificaciones aplicadas durante nuestra intervención pueden mejorar el desarrollo de destrezas infantiles y proporcionar más oportunidades para desarrollar el revés.
Palabras clave: Entrenamiento basado en las limitaciones, mini tenis, intervención, revés

Recibido: 29 Agosto 2018

Aceptado: 2 Octubre 2018

Autor correspondiente: Anna

Fitzpatrick, Universidad de

Sheffield Hallam, Inglaterra

Email:

Anna.Fitzpatrick@shu.ac.uk

\section{INTRODUCCIÓN}

Las versiones modificadas del tenis, como mini tennis y tennis play and stay, fueron diseñadas para mejorar el desarrollo de habilidades en los niños y para reducir la velocidad del juego, de modo que las acciones de los niños sean similares a las que necesitan los adultos para el tenis estándar (Buszard y cols., 2016). A pesar de que una evidencia considerable sugiere que estas versiones modificadas del tenis realmente facilitan el desarrollo táctico y técnico de los niños (ej. Larson y Guggenheimer, 2013; Timmerman y cols, 2015), los argumentos que dicen que permiten que los niños realicen acciones que se asemejen a las del tenis estándar, son bastante especulativos. Fitzpatrick y cols. (2017) investigaron este concepto dentro del mini tennis (MT); si bien el MT originó peloteos más largos con menos errores que el tenis estándar, según el análisis, los jugadores de MT ejecutaron considerablemente más golpes de derecha que de revés durante el juego de partidos (relación. 2:1). Por otro lado, la relación entre golpes de derecha y de revés realizados en el tenis estándar se acerca a 1:1 (Reid, Morgan y Whiteside, 2016). Además, la diferencia entre los golpes de derecha y de revés durante los partidos de MT puede ser aún mayor dentro de las sesiones de entrenamiento de niños, Farrow y Reid (2010) encontraron una relación de aproximadamente 6:1 a favor del golpe de derecha. Se ha señalado que tal asimetría entre el golpe de derecha y el de revés puede llevar a un desequilibrio




de destrezas con el tiempo, posiblemente en perjuicio del desarrollo del rendimiento de los niños (Fitzpatrick y cols., 2017). Por ejemplo, si los jugadores de MT no reciben suficientes oportunidades de ejecutar reveses, el golpe puede no desarrollarse correctamente, permitiendo la aparición de debilidades, que los adversarios podrían explotar. Hemos implementado aquí una intervención de 8 semanas para el entrenamiento del MT Rojo, para mejorar el desarrollo de las destrezas de los niños, disminuyendo simultáneamente la diferencia entre el rendimiento en el golpe de derecha y el de revés.

\section{MÉTODO}

\section{Participantes}

Se asignaron 16 niños a cada uno de los dos grupos de forma aleatoria; control ( $n=8$, edad $7.2 \pm 0.6$ años, experiencia en el juego de tenis $1.9 \pm 0.6$ años) y experimental ( $n=8$, edad 7.4 \pm 0.4 años, experiencia en el juego de tenis $2.1 \pm 0.6$ años). Todos los niños eran diestros y ejecutaban el revés a dos manos.

\section{Procedimiento}

Las pruebas pre y post comprendían dos elementos: el juego de partidos y la prueba de destrezas específicas para el tenis (TSST, por sus siglas en inglés).

\section{Pre-test:: juego de partidos}

Se filmó a cada jugador completando tres partidos estándar de MT Rojo 'el primero en llegar a 10 puntos' (LTA, 2017), contra tres adversarios asignados aleatoriamente.

\section{Pre-test TSST}

Los jugadores intentan mantener tres peloteos con el entrenador, lo más largos posibles. La media de longitud del peloteo de los tres intentos produce un "puntaje de rendimiento en el peloteo". Además, dos entrenadores de Nivel 3 de la LTA evaluaron cualitativamente cuatro aspectos de la producción de los golpes, para golpe de derecha, y revés respectivamente: movimiento $26^{\circ}$ Año, Número 76, Diciembre 201811 de la pelota, movimiento de preparación, impacto de la pelota/ terminación, y recuperación, utilizando una escala de 7 puntos (Farrow y Reid, 2010). Se calcularon los cuatro puntajes de los golpes de derecha y de revés respectivamente, obteniendo un máximo "puntaje lograble de competencia técnica" de 28 puntos por golpe.

\section{Intervención}

Ambos grupos asistieron a un programa de entrenamiento de MT de 8 semanas ( 1 hora por semana). Ambos grupos recibieron la enseñanza del mismo entrenador, y realizaron las mismas actividades, pero se modificó el entorno de aprendizaje del grupo experimental. (Ver Figura 1).

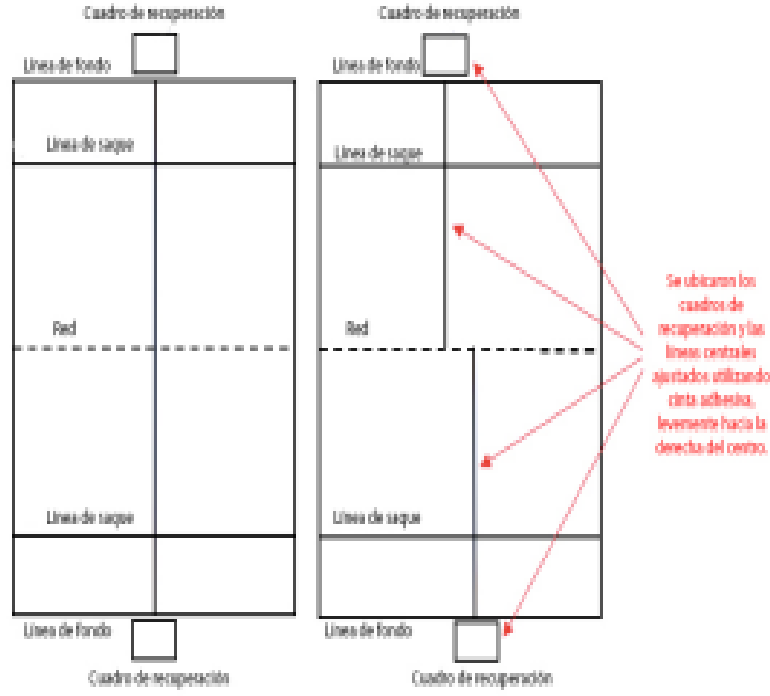

Figura 1. Ubicación del cuadro de recupención y de la línea central para el grupo control (zquilerda) y para el grupo experimental (derecha).

Los jugadores en el grupo experimental debían intentar jugar un revés si la pelota botaba a la izquierda de la línea central ajustada (Hopper, 2011), y volver al cuadro de recuperación después de cada golpe (Bryant, 2012). Además durante las actividades basadas en puntos del grupo experimental, el entrenador otorgaba puntos extra (un incentivo agregado) si el jugador presionaba a su adversario utilizando su revés (Hopper, 2011).

Pos-test

Todos los jugadores fueron filmados completando los tres partidos de MT Rojo, contra los mismos tres adversarios que en el pre-test y repitieron el TSST.

Análisis de los datos

Los datos de los vídeos de los partidos se codificaron utilizando un sistema de análisis notacional personalizado (fiabilidad entre evaluadores $k=0.95$ ). Posteriormente se calcularon las variables mostradas en la Tabla 1 (ver lista completa en Fitzpatrick y cols., 2018); los valores de la eficiencia técnica TSST y de rendimiento en el peloteo se redujeron a valores medios.

\begin{tabular}{|l|l|}
\hline Variable dependiente & Ecuación \\
\hline Golpe de derecha \% & $\begin{array}{l}\text { (N. de golpes de derecha/ total de tiros } \\
\text { jugados tras el servicio) X 100 }\end{array}$ \\
\hline Golpe de revés \% & $\begin{array}{l}\text { (N. de golpes de revés/ total de tiros } \\
\text { jugados tras el servicio) x 100 }\end{array}$ \\
\hline
\end{tabular}

Tabla 1. Variables paro el jueqo de partidos. 
Se hizo análisis de varianza de diseño mixto de dos vías (ANOVA) (condición de la práctica por tiempo) para investigar los efectos de la intervención. No se detectaron diferencias estadísticas entre el número total de tiros ejecutados por cada grupo durante la intervención, por lo tanto, los efectos de la intervención no fueron atribuibles a las diferencias en la frecuencia de las acciones realizadas.

\section{RESULTADOS}

Aquí se presentan los resultados clave (para todos los resultados, ver Fitzpatrick y cols., 2018).

\section{Tipo de tiros en el juego de partidas}

La Figura 2 muestra que el porcentaje de reveses ejecutados por el grupo experimental aumentó un $17,0 \%$ tras la intervención, el porcentaje del grupo control disminuyó 1,8\%. El porcentaje de golpes de derecha ejecutados por el grupo experimental disminuyó un 17,3\% tras la intervención, mientras que el porcentaje del grupo control no presentó cambios.

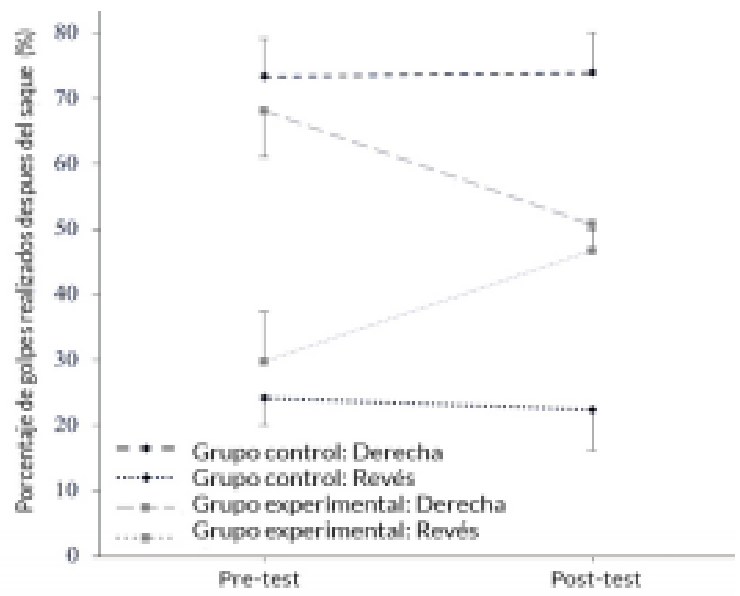

Figura 2. Porcentaje de golpes de derecha y de revés realizados por cac grupo.

Puntuación de competencia técnica TSST y rendimiento en el peloteo

La Figura 3 muestra que la competencia técnica del golpe de derecha y revés del grupo experimental mejoró en 3,3 puntos y 4,0 puntos respectivamente, tras la intervención. El grupo control mejoró menos, 1,5 puntos en el golpe de derecha, y 0,8 en el golpe de revés respectivamente. Además, el puntaje en el peloteo del grupo experimental se incrementó en 7,6 golpes después de la intervención (de 16,2 a 23,8 tiros); los del grupo control incrementaron en 2,9 golpes (de 14,3 a 17,2 tiros).

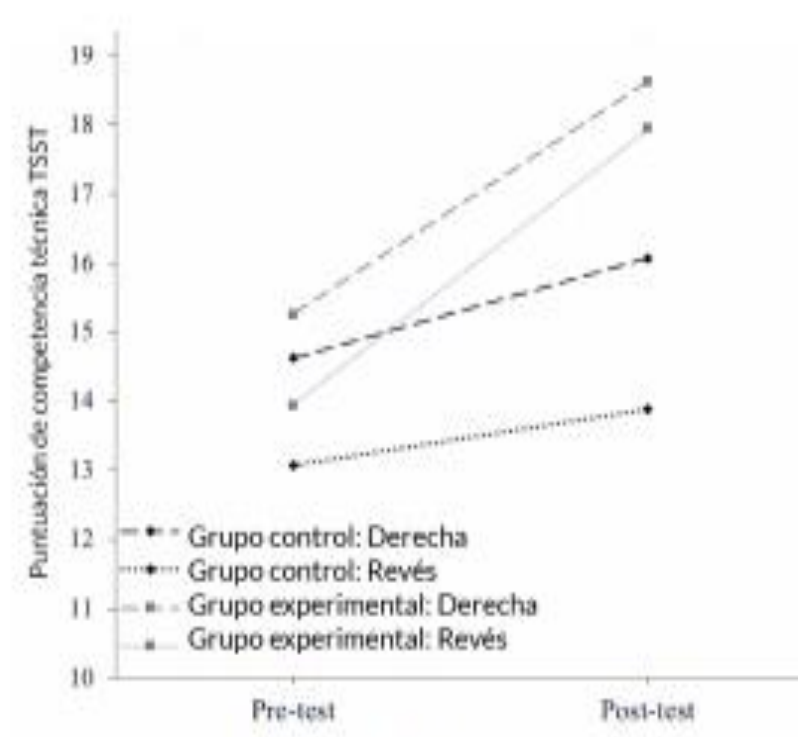

Figura 3. Puntuación de competencia técnico T55T.

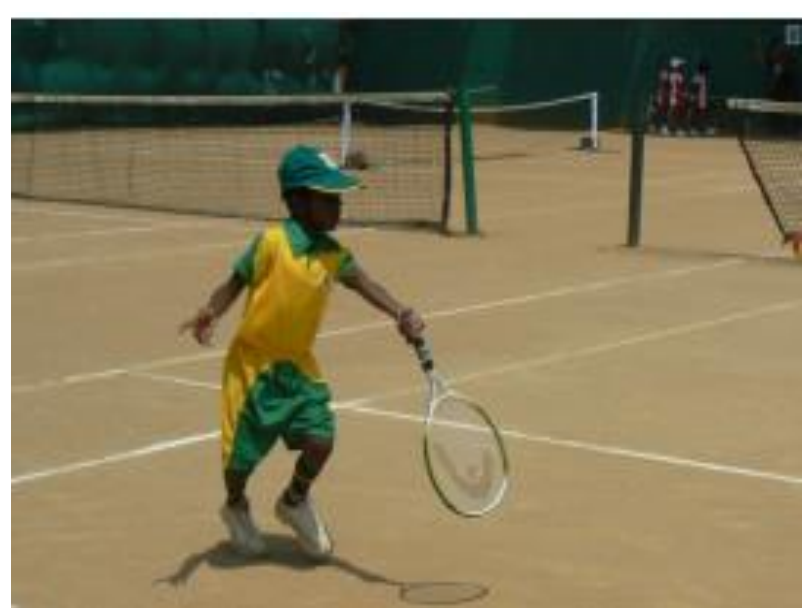

\section{DISCUSIÓN}

Los datos de los partidos pre-test replicaron la asimetría encontrada en Fitzpatrick y cols. (2017), con ambos grupos realizando un número desproporcionadamente alto de golpes de derecha comparado con los de revés. Durante el post-test, el grupo experimental demostró mayor simetría (46,7\% reveses, 50,8\% golpes de derecha), comparados con la continua asimetría del grupo control (74,0\% derechas, $22,4 \%$ reveses). Los valores post-test del grupo experimental se correspondieron estrechamente a los ratios entre golpe de derecha y revés observados en el tenis estándar (cerca de 1:1). Los ratios observados en el tenis estándar demuestran que es clave que los jugadores desarrollen ambos golpes de fondo, si desean lograr una exitosa transición a través de las etapas del tenis. 
EI MT Rojo permite a los jugadores tiempo suficiente para moverse alrededor de la pelota y realizar un golpe de derecha, cuando sería más apropiado un golpe de revés (Fitzpatrick y cols., 2017). Sin embargo, este es un movimiento ineficiente (utiliza más tiempo y energía), es improbable que se realice una técnica óptima (Hodgkinson, 2015), y perjudicial para los movimientos de recuperación del jugador (Hughes y Moore, 1998). Posicionando el cuadro de recuperación del grupo experimental ligeramente hacia el golpe de derecha en la cancha, se incrementaba la distancia que los jugadores debían desplazarse para posicionarse hacia la izquierda de la pelota y jugar un golpe de derecha, haciendo que esta conducta sea menos probable. En cambio, nuestras modificaciones estimularon a los jugadores a adaptarse y explorar diferentes soluciones (o sea, jugar un revés), lo que puede facilitar una técnica más funcional.

Así, la competencia técnica del golpe de derecha del grupo experimental mejoró más que la del grupo control. Sin embargo, es interesante destacar que también mejoró la técnica del golpe de derecha del grupo experimental más que en el grupo control, a pesar de golpear menos golpes de derecha durante el juego de partidos. Esto indica que, tras la intervención, el grupo experimental eligió jugar cada golpe sólo cuando correspondía, y por lo tanto, mostró una más técnica funcional. Por el contrario, el grupo control continuó intentando moverse alrededor de la pelota y realizar un golpe de derecha cuando hubiera sido más apropiado un revés, entonces, aunque el grupo control realizó más golpes de derecha, generalmente la técnica obtenida fue frecuentemente deficiente. Parece evidente que las modificaciones introducidas en el juego, han influido en el movimiento de los jugadores hacia la pelota y en la recuperación, entonces, parece que la intervención ha mejorado el movimiento del grupo experimental en la cancha, al igual que su técnica para el movimiento de preparación.

La puntuación de rendimiento en peloteo del grupo experimental (o sea, el peloteo con el entrenador) también mejoró más que el del grupo control, sin embargo, ambos grupos demostraron mejoras similares en la duración del peloteo durante la disputa de partidos (es decir en peloteos con sus pares) (ver Fitzpatrick y cols., 2018). Para los niños es más fácil pelotear con un entrenador, quien controla la dirección y el ritmo de cada golpe. De este modo, parecería que la intervención mejora suficientemente la habilidad de peloteo en el grupo experimental como para dar origen a peloteos con el entrenador, pero no lo suficiente como para replicarlo durante el juego de partidos con sus pares.

\section{CONCLUSIÓN}

Los resultados indicaron que nuestra intervención disminuyó de manera efectiva la asimetría existente entre la ejecución del golpe de derecha y el de revés durante el juego de partidos de los niños. Simultáneamente, el grupo experimental demostró mejores habilidades de peloteo haciéndolo con el entrenador, y mejor competencia técnica, ofreciendo gran apoyo a las modificaciones aquí aplicadas. Los entrenadores, si lo desean, pueden implementar modificaciones similares durante las sesiones de entrenamiento, para mejorar el desarrollo de las destrezas de los niños y reducir la disparidad entre los porcentajes de golpe de derecha y de revés jugados normalmente.

\section{REFERENCIAS}

Bryant J. E. (2012). Game/set/match: a tennis guide. (8th ed.). Boston: Cengage Learning

Buszard, T., Reid, M., Masters, R., \& Farrow, D. (2016). Scaling the equipment and play area in children's sport to improve motor skill acquisition: a systematic review. Sports Medicine, https://doi.org/10.1007/s40279-015-0452-2

Farrow, D. \& Reid, M. (2010). The effect of equipment scaling on the skill acquisition of beginning tennis players. Journal of Sports Sciences, 28, 723-732. https://doi.org/10.1080/02640411003770238

Fitzpatrick, A., Davids, K., \& Stone, J. A. (2017). Effects of Lawn Tennis Association Mini Tennis as task constraints on children's matchplay characteristics. Journal of Sports Sciences, 35(22), 2204 2210. https://doi.org/10.1080/02640414.2016.1261179

Fitzpatrick, A., Davids, K., \& Stone, J. A. (2018). Effects of scaling task constraints on emergent behaviours in children's racquet sports performance. Human Movement Science, 58, 80-87 https://doi.org/10.1016/j.humov.2018.01.007

Hodgkinson, M. (2015). Game, set and match: secret weapons of the world's top tennis players. Londres: Bloomsbury

Hopper, T. (2011). Game-as-teacher: modification by adaptation in learning through game-play. Asia-Pacific Journal of Health, Sport and Physical Education, 2, 3-21. https://doi.org/10.1080/18377122.2011.9730348

Hughes, M. \& Moore, P. (1998). Movement analysis of elite level male 'serve and volley' tennis players. In A. Lees, I. Maynard, M. Hughes and T. Reilly (eds.), Science and racket sports II. (pp. 254-259) London: E \& FN Spon

Larson, E J. \& Guggenheimer, J D. (2013). The effects of scaling tennis equipment on the forehand groundstroke performance of children. Journal of Sports Science and Medicine, 12, 323- 331.

LTA. (2017). LTA - Mini Tennis. Retrieved from http://www3.Ita.org. uk/LTA-Mini-Tennis

Reid, M., Morgan, S., \& Whiteside, D. (2016). Matchplay characteristics of Grand Slam tennis: implications for training and conditioning. Journal of Sports Sciences, 34, 1791-1798. https://doi.org/10.1080/02640414.2016.1139161

Timmerman, E., de Water, J., Kachel, K., Reid, M., Farrow, D. \& Savelsbergh, G. (2015). The effect of equipment scaling on children's sport performance: the case for tennis. Journal of Sports Sciences, 33, 1093-1100. https://doi.org/10.1080/02640414.2014.986498 
Este texto está protegido por una licencia CreativeCommons $\underline{4.0}$.

Usted es libre para Compartir — copiar y redistribuir el material en cualquier medio o formato-y Adaptar el documento - remezclar, transformar y crear a partir del material- para cualquier propósito, incluso para fines comerciales, siempre que cumpla la condición de:

Atribución: Usted debe dar crédito a la obra original de manera adecuada, proporcionar un enlace a la licencia, e indicar si se han realizado cambios. Puede hacerlo en cualquier forma razonable, pero no de forma tal que sugiera que tiene el apoyo del licenciante o lo recibe por el uso que hace de la obra.

Resumendelicencia - Textocompletodelalicencia 